\title{
Ligand Binding Phenomena that Pertain to the Metabolic Function of Renalase
}

Brett A. Beaupre ${ }^{1}$, Joseph V. Roman ${ }^{1}$, Matthew R. Hoag ${ }^{1}$, Kathleen M. Meneely', Nicholas R. Silvaggi ${ }^{1}$, Audrey L. Lamb ${ }^{2}$ and Graham R. Moran ${ }^{1}$ *

${ }^{1}$ Department of Chemistry and Biochemistry, University of Wisconsin-Milwaukee, 3210

N. Cramer St, Milwaukee, Wisconsin 53211-3209.

${ }^{2}$ Molecular Biosciences, University of Kansas, 1200 Sunnyside Ave, Lawrence, KS 66049.

This publication was made possible by funds from National Science Foundation grants numbered CHE-1402475 (G.R.M.), CHE-1403293 (A.L.L.) and MCB-1157392 (N.R.S), National Institutes of Health grant numbered K02 Al093675 from the National Institute for Allergy and Infectious Disease (A.L.L.) and by a UWM Research Growth Initiative Grant (G.R.M.).

*To whom correspondence should be addressed:

Ph: (414) 9400059

Fax: (414) 2295530

Email: moran@uwm.edu 


\section{Abbreviations}

ADP, adenosine diphosphate; ADP-ribose, adenosine diphosphate ribose; AMP, adenosine monophosphate; $\beta-\mathrm{NAD}^{+}$, oxidized nicotinamide adenine dinucleotide; $\beta$ $\mathrm{NADP}^{+}$, oxidized nicotinamide adenine dinucleotide phosphate; $\beta$-NADPH, reduced nicotinamide adenine dinucleotide phosphate; FAD, flavin adenine dinucleotide; HPLC, high performance liquid chromatography; HsRen, renalase from $H$. sapiens; NMN, nicotinamide mononucleotide; NR, nicotinamide riboside; PpRen, renalase from $P$. phaseolicola, 6DHNAD, 6-dihydronicotinamide adenine dinucleotide; 2DHNAD, 2dihydronicotinamide adenine dinucleotide; 6DHNADP, 6-dihydronicotinamide adenine dinucleotide phosphate; 2DHNADP, 2-dihydronicotinamide adenine dinucleotide phosphate; 4DHNMN, 4-dihydronicotinamide mononucleotide; 6DHNMN, 6dihyronicotinamide mononucleotide; 2DHNMN, 2-dihydronicotinamide mononucleotide; 4DHNR, 4-dihydronicotinamide riboside; 6DHNR, 6dihydornicotinamide riboside. 


\section{Abstract}

Renalase catalyzes the oxidation of isomers of $\beta-\mathrm{NAD}(\mathrm{P}) \mathrm{H}$ that carry the hydride in the 2 or 6 positions of the nicotinamide base to form $\beta-N A D(P)^{+}$. This activity is thought to alleviate inhibition of multiple $\beta-N A D(P)$-dependent enzymes of primary and secondary metabolism by these isomers. Here we present evidence for a variety of ligand binding phenomena relevant to the function of renalase. We offer evidence of the potential for primary metabolism inhibition with structures of malate dehydrogenase and lactate dehydrogenase bound to the 6-dihydroNAD isomer. The previously observed preference of renalase from Pseudomonas for NAD-derived substrates over those derived from NADP is accounted for by the structure of the enzyme in complex with NADPH. We also show that nicotinamide nucleosides and mononucloetides reduced in the 2- and 6-positions are renalase substrates, but bind weakly. A seven-fold enhancement of acquisition $\left(k_{\text {red }} / K_{d}\right)$ for 6 -dihydronicotinamide riboside was observed for human renalase in the presence of ADP. However, generally the addition of complement ligands, ADP for mononucloetide or AMP for nucleoside substrates, did not enhance the reductive half-reaction. Non-substrate nicotinamide nucleosides or nucleotides bind weakly suggesting that only $\beta$-NADH and $\beta$-NADPH compete with dinucleotide substrates for access to the active site. 


\section{Introduction}

Renalase is an FAD-dependent oxidase that catalytically oxidizes two isomeric forms of $\beta-N A D(P) H$ to form $\beta-N A D(P)^{+}$and $\mathrm{H}_{2} \mathrm{O}_{2}$ (Scheme 1). These isomers are presumed to occur when $\beta-\mathrm{NAD}(\mathrm{P})^{+}$is reduced non-specifically forming, in addition to the native $\beta-N A D(P) H$ (4-dihydroNAD(P)), 2-dihydroNAD(P) (2DHNAD(P)) and 6dihydroNAD(P) (6DHNAD(P)) $[1,2]$. Both 2DHNAD and 6DHNAD have been shown to be highly inhibitory to specific primary metabolism dehydrogenases [2]. This suggests that renalase has an intracellular metabolic house-keeping function that alleviates metabolic suppression by these isomers. This proposal differs starkly from the consensus view that renalase is a mammalian serum borne protein that is associated with an array of aberrant physiological conditions. The enzyme was originally claimed to be a kidneyderived hormone that down-modulates vascular tone in animals by the oxidation of catecholamines[3-7] and then later to be a cytokine that ameliorates myocardial damage resulting from an ischemic event[8]. More recently it has been said to be a suppressor of pancreatic cancer and/or an exacerbatory factor for melanoma [4, 9-12]. Here we present the first structures of 6-DHNAD in complex with malate dehydrogenase and lactate dehydrogenase, confirming our assertion that $\beta-N A D(P) H$ [dihydronicotinamide] isomers are detrimental to normal metabolic activity and reasserting the verified catalytic function of renalase. These structures show that 6DHNAD occupies the $\beta$-NADH-binding site occluding the association of the native nicotinamide substrate. 
With a modest two-electron reduction potential of $-320 \mathrm{mV}$, the potential for non-specific redox reactions of $\beta-N A D(P)^{+}$that form toxic $\beta-N A D(P) H$ isomers exists in all living systems. It is therefore reasonable to expect that an intracellular detoxification activity such renalase would be found in multiple kingdoms of life. However, homology searches based on the human renalase amino acid sequence return almost exclusively homologs from animalia. The structure of human renalase (isoform 1; HsRen) was solved in 2011 by the Aliverti group who noted that the renalase structural topology was common to numerous redox active flavoproteins [13]. In this structure the open active site was observed to have only a small number of conserved amino acids indicating that sequence alignments based on overall alignment scores (E-values) may not detect this motif and will not necessarily identify distant forms of renalase. We have recently characterized a renalase from a Pseudomonad (19\% identity to Human)[14]. As part of this study we solved the crystal structures of this form of renalase (PpRen) in complex with $\beta$-NADH (PDB ID 4ZCC, $2.1 \AA$; a fascimile of the ES complex) and $\beta-N A D^{+}$(PDB ID 4ZCD, $1.7 \AA$; the EP complex). These structures revealed that the si face of the FAD isoalloxazine is closely associated with the inner surface of the active site offering the re face for interaction with the substrate nicotinamide base. The few conserved residues that line the dihydronicotinamide-binding cavity are: PpRen H232 (HsRen H245), W267 (HsRen W288) and R280, which is apparently equivalent to HsRen R193, as both residues offer their guanidino group to a similar location in the active site. In both proteins, the active site cavity forms one end of an extended cleft to which $\beta$-NAD(P)derived substrate(s) associate (Figure 1). Within this cleft are multiple hydrogen bonds 
and charge pairing interactions that in the Pseudomonas enzyme form a rather symmetrical set of interactions with respect to each nucleotide half of the substrate. However, the majority ( 8 of 11 ) form hydrogen bonding and/or charge pairing interactions with the pyrophosphate moiety of the substrate/product. Only one direct contact is observed for the nicotinamide amide and two for the 6 amino group of the adenine base with no hydrogen bonds from the protein engaging any of the hydroxyl groups of either ribose. This arrangement raises interesting questions about what part of the molecule contributes to the binding energy given that renalase must function in an environment in which non-substrate mono- and dinucleotides predominate. Using truncated forms of the substrate we offer evidence that, at least for human renalase, binding of the pyrophosphate moiety of the substrate has a primary contribution to stabilizing the pre-reduction $\mathrm{E} \bullet \mathrm{S}$ complex.

The substrate/product binding pose exposes one face of the ligand to direct interactions with solvent (Figure 1). Both the human (HsRen) and bacterial (PpRen) forms of renalase exhibit a preference for substrates derived from $\beta-N A D^{+}$over those derived from $\beta-\mathrm{NADP}^{+}$and this bias is notably more pronounced in the bacterial enzyme [14]. While this specificity bias is consistent with renalase serving to preferentially detoxify the inhibitory effect that 2DHNAD and 6DHNAD would exert on primary metabolism enzymes, the structural basis for this selectivity was hitherto unknown. In this study we present the structure of renalase from Pseudomonas phaseolicola in complex with $\beta$-NADPH and evaluate the influence of the ribose 2-phospho group in regard to the observed substrate specificity. 


\section{Materials and Methods}

Materials. Dibasic potassium phosphate and sodium phosphate, monobasic potassium phosphate and sodium chloride were obtained from ACROS. $\beta$-NADH (disodium salt, trihydrate) was obtained from Amresco. $\beta-N A D^{+}, \beta-N A D P^{+}$, nicotinamide mononucleotide (NMN), ADP-ribose, methyl nicotinamide and nicotinic acid were purchased from Sigma. Nicotinamide riboside (NR) was purchased from High Performance Nutrition. Renalases from H. sapiens (HsRen) and P. phaseolicola (PpRen) were expressed and purified according to previously published methods[14, 15]. Old yellow enzyme was prepared as previously described[2]. 6DHNAD and 6DHNADP were prepared and purified as described[2].

Preparation and analysis of reduced forms of $N R$ and NMN. To establish whether nucleoside and nucleotide forms of 2-dihydro- and 6-dihydronicotinamides were substrates for renalase, $0.9 \mathrm{~mL}$ of a $300 \mu \mathrm{M}$ solution of oxidized nicotinamide riboside $\left(\mathrm{NR}^{+}\right)$or nicotinamide mononucleotide $\left(\mathrm{NMN}^{+}\right)$was combined with $100 \mu \mathrm{L}$ of a $3 \mathrm{mM}$ solution of sodium borohydride in $100 \mathrm{mM}$ potassium phosphate at $\mathrm{pH}$ 7.5. This resulted in instantaneous generation of the three reduced forms of each parent compound (2-, 4- and 6-dihydro). Each of these mixtures was divided in two and 12.5 $\mu \mathrm{M}$ renalase (final) was added to one sample, while the other served both as unreacted and sample stability controls. All samples were incubated for $10 \mathrm{~min}$ prior to ultrafiltration using a $0.5 \mathrm{~mL}$ Amicon $10 \mathrm{kDa}$ centrifugal filter to remove renalase. $50 \mu \mathrm{L}$ of each filtrate was loaded onto an analytical Xbridge Peptide BEH C18 column (3.5 $\mu \mathrm{M}$, 
$4.6 \times 150 \mathrm{~mm}$ ) run isocratically at $1.5 \mathrm{~mL} / \mathrm{min}$ in $100 \mathrm{mM}$ potassium phosphate at $\mathrm{pH} 7.5$ with a Waters 600 pump coupled to a Waters 2487 dual wavelength detector, detecting simultaneously at 260 and $370 \mathrm{~nm}$. The chromatographs obtained from samples to which renalase was added were corrected for the dilution.

For preparation of the 4DH forms of $\mathrm{NR}$ and $\mathrm{NMN}$, sodium dithionite was used as a reductant in place of sodium borohydride [2]. 4DHNR and 4DHNMN were prepared by semi-preparative HPLC. The dithionite reduced mixtures were loaded onto a semi preparative Xbridge Prep C18 OBD column ( $5 \mu \mathrm{M}, 19 \times 250 \mathrm{~mm})$ run isocratically at 10 $\mathrm{mL} / \mathrm{min}$ in $100 \mathrm{mM}$ potassium phosphate buffer, pH 7.5 using a Waters 600 pump coupled to a Waters 2487 dual wavelength detector, detecting simultaneously at 260 and $340 \mathrm{~nm}$. 4DHNR and 4DHNMN samples were frozen on dry ice during multiple collection cycles and then thawed and loaded onto a 35 cc Sep Pak (Waters) C18 cartridge equilibrated in $100 \mathrm{mM}$ potassium phosphate $\mathrm{pH}$ 7.5. The bound sample and was eluted with water into $3 \mathrm{~mL}$ fractions. The fractions that contained the desalted 4DHNR or 4DHNMN were pooled and immediately lyophylized. Once dry, the sample was dissolved in $6 \mathrm{~mL}$ of cold water and stored at $-80{ }^{\circ} \mathrm{C}$.

Spectrophotometric quantification of NAD analogs. Molar extinction coefficients for 2-, 4- and $6 \mathrm{DHNMN}$ and 2-, 4- and 6DHNR were determined by reducing a $4 \mathrm{~mL}$ solution containing $\sim 20 \mathrm{mM} \mathrm{NMN}^{+}$or $\mathrm{NR}^{+}$in $100 \mathrm{mM}$ potassium phosphate buffer, pH 7.5 with 1 $\mathrm{mL}$ of $\sim 66 \mathrm{mM}$ sodium borohydride solution prepared in $30 \mathrm{mM}$ potassium phosphate buffer, $\mathrm{pH}$ 11. The resulting $5 \mathrm{~mL}$ solution containing all three reduced isomers was 
separated by semi-preparative HPLC as described above. Peaks corresponding to each of the reduced isomers were collected. Both 4- and 6-dihydro forms were frozen on dry ice while the less stable 2-dihydro isomers were used immediately. The concentration of each isomer was determined by the oxidase activities of renalase (2- and 6-dihydro forms) or old-yellow enzyme (4-dihydro form) using a Hansatech dioxygen electrode. Briefly, $1.6 \mathrm{~mL}$ of each sample collected was divided in two, where one half was used to obtain an absorption spectrum and the other was loaded into the oxygen electrode reaction vessel and allowed to react with either $10 \mu \mathrm{M}$ renalase (2- and 6-dihydro NMN or NR) or $20 \mu \mathrm{M}$ old yellow enzyme (4-dihydro NMN or NR). Based on each enzyme's reaction stoichiometry, the amount of oxygen consumed $(\mu \mathrm{M})$ defined the concentration of the dihydronicotinamide substrate. This measurement was made three times for each isomer and the results were averaged after correction for the dilution that occurred with the addition of enzyme.

Reductive half-reactions with 2- and 6DHNMN and 2- and 6DHNR. Exclusion of dioxygen allows for observation of only the reductive half-reaction of renalase. Rate constants for reduction were determined using HsRen and PpRen as previously described $[2,14]$. The post mixing concentration of HsRen was 6-11 $\mu \mathrm{M}$ and 2-5.5 $\mu \mathrm{M}$ for PpRen. Reduction of the renalase flavin cofactor was monitored at $458 \mathrm{~nm}$ for HsRen and $450 \mathrm{~nm}$ for PpRen. All half-reactions were carried out in 20 mM HEPES buffer, $\mathrm{pH}$ 7.5. Reactions with 2- and 6DHNR as substrates required the addition of the detergent octyl-beta-glucoside ( $2 \mathrm{mM}$ final) in the tonometer to prevent excessive enzyme precipitation during the reaction. 
The reduction process was fit to equation 1 to obtain $k_{\text {obs }}$ values. In this equation $\Delta \mathrm{A}$ is the amplitude associated with the change in the enzyme's flavin absorption spectrum as it is reduced, $k_{\text {obs }}$ is the observed rate constant for reduction of the flavin cofactor and $C$ is the absorbance at infinite time. The $k_{\text {obs }}$ values were then plotted against substrate concentration and fit to Equation 2 where the slope of the line gave $k_{\text {red }} / K_{d}$ as a measure of the enzyme's substrate acquisition capacity, where $k_{\text {red }}$ is the limit for the rate constant for reduction and $\mathrm{K}_{\mathrm{d}}$ is the dissociation constant for the substrate, $\mathrm{S}$.

Equation $1 \quad A_{458}=\Delta A\left(e^{-k_{o b s} t}\right)+C$

Equation $2 \quad k_{o b s}=\left(k_{\text {red }} / K_{d}\right)[S]$

The available concentration of 2DHNR and 2DHNMN was limited by instability. Samples could not be pooled and further purified, but were instead collected directly from the HPLC into a glass syringe containing D-glucose ( $1 \mathrm{mM}$ final) and sparged with argon for 3 minutes. Glucose oxidase $(10 \mu \mathrm{L}, 1 \mathrm{U} / \mu \mathrm{L})$ was added to the glass syringe immediately before mounting onto the stopped flow spectrophotometer to consume dissolved dioxygen.

Reductive half reactions were also undertaken in the presence of counterpart ligands. 2- and 6DHNMN and 2- and 6DHNR were used as substrates for renalase in the presence of AMP for NMN and ADP for NR. These experiments used double mixing stopped-flow spectrophotometry where renalase (HsRen, $10 \mu \mathrm{M}$ and PpRen, $5 \mu \mathrm{M}$ ) was 
prepared anaerobically in 20 mM HEPES buffer, $\mathrm{pH} 7.5$ and mixed with AMP or ADP and allowed to age for $100 \mathrm{~ms}$ before being mixed/reacted with 2- or 6DHNMN and 2- or 6DHNR respectively. Data were collected and analyzed as described above. Octyl-betaglucoside $(2 \mathrm{mM})$ was added to the enzyme solution for reactions containing 2- and 6DHNR.

Inhibition of human renalase by $\beta-N A D(P)$, non-substrate analogs and fragments. The $50 \%$ inhibitory concentration $\left(I C_{50}\right)$ of NAD fragments: ADP, ADP-ribose, AMP, nicotinamide, methyl nicotinamide, $\mathrm{NR}^{+}, \mathrm{NMN}^{+}, 4 \mathrm{DHNR}$, and $4 \mathrm{DHNMN}$, were estimated by observing the renalase reductive half reaction in the absence of dioxygen and in the presence of a 6DHNAD concentration that was equal to the measured dissociation constant for that substrate $(173 \mu \mathrm{M})[2]$. Renalase $(\sim 4-6 \mu \mathrm{M})$ in $2 \mathrm{X}$ PBS buffer containing $1 \mathrm{mM}$ D-glucose was added to the main chamber of a tonometer and glucose oxidase (25 $\mu \mathrm{L}, 1 \mathrm{U} / \mu \mathrm{L}$ ) was added to the side arm prior to assembly and was kept at $4{ }^{\circ} \mathrm{C}$. The tonometer was assembled and made anaerobic by exchanging argon for dissolved oxygen using partial vacuum followed by equilibration with high purity argon gas (5 psi) that was passed through an Alltech oxygen-reactive cartridge and sparged through anaerobic water. This process was repeated for 45 cycles where after every 3 cycles the sample was agitated during the introduction of argon gas to enhance the exchange of dissolved gases. The glucose oxidase and D-glucose/renalase solutions were combined and the tonometer was mounted on a Hitech Scientific (now TgK) DX2 stopped-flow spectrophotometer that had been scrubbed of dioxygen for $>12 \mathrm{hrs}$ using an anaerobic 
solution of $50 \mathrm{mM} \mathrm{D-glucose}$ and $15 \mathrm{U} / \mathrm{mL}$ glucose oxidase in $20 \mathrm{mM}$ HEPES buffer, $\mathrm{pH}$ 7.0. Concentrated 6DHNAD was aliquoted to yield $173 \mu \mathrm{M}$ after the second mixing step (four-fold dilution) and stored at $-80{ }^{\circ} \mathrm{C}$ to prevent decomposition. Each aliquot was thawed and diluted to $3 \mathrm{~mL}$ in water containing $1 \mathrm{mM}$ D-glucose. The solution containing 6DHNAD was transferred to a glass syringe and made anaerobic by sparging with high purity argon gas for 4 minutes. Glucose oxidase (10 $\mu \mathrm{L}, 1 \mathrm{U} / \mu \mathrm{L})$ was added to the syringe immediately prior to capture and mounting onto the stopped-flow spectrophotometer. Potential inhibitory ligands were prepared in 2X PBS with 1 mM Dglucose, transferred into a glass syringe and made anaerobic as described above for 6DHNAD. The renalase solution was mixed first with the NAD analog (or fragment) solution and allowed to age for $100 \mathrm{~ms}$ before being mixed with the substrate (6DHNAD) solution. Reduction of the renalase flavin cofactor was observed at $458 \mathrm{~nm}$. The data were fit to a single exponential decay according to equation 1 using Kinetic Studio software (TgK Ltd). The dependence of the observed rate constant on the concentration of the analog was fit to Equation 3 to determine the $\mathrm{IC}_{50}$ value for each. In this equation $k_{\max }$ is the rate constant for reduction in the absence of inhibitory ligand and $k_{\min }$ is the observed rate constant with saturating inhibitory ligand (this value was manually set to zero for cases in which the inhibitory ligand had a solubility limit lower than the concentration required to saturate the enzyme).

Equation $3 \quad v=k_{\min }+\frac{k_{\max }-k_{\min }}{\left(1+\left(\frac{[6 D H N A D]}{K_{i}}\right)\right.}$ 
Crystallization, structure determination, and model refinement of the PpRen $\beta$-NADPH complex. Crystallization conditions for PpRen were those identified initially by the North East Structural Genomics Consortium that yielded the unliganded enzyme structure, PDB ID 3KKJ: $2 \mathrm{M}$ sodium formate, $100 \mathrm{mM}$ sodium acetate $\mathrm{pH} 4.6$ at $20^{\circ} \mathrm{C}$. Diffractionquality crystals were obtained by the hanging drop vapor diffusion method. The droplet was formed from $1 \mu \mathrm{L}$ of the well solution and $1 \mu \mathrm{L}$ of PpRen $(104 \mu \mathrm{M})$. Crystals grew within 1-2 days to dimensions of $\sim 200 \mu \mathrm{m} \times 50 \mu \mathrm{m} \times 10 \mu \mathrm{m}$. Individual crystals were lifted from the drops using nylon cryo-loops, and soaked for 1-3 minutes in $2.5 \mathrm{M}$ sodium formate, $125 \mathrm{mM}$ sodium acetate $\mathrm{pH} 4.7$ with $10 \%$ glycerol and $40 \mathrm{mM} \beta$ NADPH. The loop-mounted, cryo-solution and crystal were cooled to form a glass by immersion in liquid nitrogen. X-ray diffraction data to $2.1 \AA$ for the PpRen• $\beta$-NADPH complex were collected at beamline 21-ID-F of the Life Science Collaborative Access Team (LS-CAT) at the Advanced Photon Source (APS) at a wavelength of $0.97872 \AA$ at $100 \mathrm{~K}$. The crystal was rotated through $190^{\circ}$, with an oscillation angle of $0.4^{\circ}$. The exposure time per frame was $0.75 \mathrm{~s}$ with $0 \%$ attenuation. The crystal to detector distance was $220.2 \mathrm{~mm}$. Data were indexed and scaled with HKL2000 [16] in space group $\mathrm{P} 2{ }_{1}$ with unit cell dimensions $\mathrm{a}=63.4 \AA \mathrm{A}=71.0 \AA$, $\mathrm{c}=74.8 \AA$, and $\beta=107.8^{\circ}$.

The structure of PpRen $\beta$-NADPH was determined by molecular replacement in PHASER[17] with a search model derived from chain A of the P. phaseolicola renalase $\bullet \beta$ NADH structure (PDB ID 4ZCC)[14] with all non-protein atoms removed and all B-factors set to $20.0 \AA^{2}$. After iterative cycles of manual model building in COOT[18] and 
maximum likelihood based refinement using the PHENIX package (phenix.refine)[19], ordered solvent molecules were added automatically in phenix.refine and culled manually in COOT. After adding solvent atoms, the FAD cofactor and $\beta$-NADPH were added to the model. During the last rounds of refinement, hydrogen atoms were added to the model using phenix.reduce[20] to improve the stereochemistry of the model. Positions of $\mathrm{H}$ atoms were refined using the riding model with a global B-factor. Regions of each model to be used in translation-libration-screw (TLS) refinement were identified using phenix.find_tls_groups and the TLS parameters were refined in phenix.refine. Once the refinement converged, the model was validated using the tools implemented in COOT and PHENIX[21]'[22]. Side chains with poor or missing electron density were modeled in favored rotameric conformations. The B-factors were allowed to refine without additional restraints, and the occupancies were held to 1.0. Data collection and model refinement statistics are listed in Table 1.

Structure determination, and model refinement of the MDH•6DHNAD and $L D H \bullet 6 D H N A D$ complexes. E. coli malate dehydrogenase $(\mathrm{MDH})$ was prepared as previously described[2]. MDH crystal growth was carried out by the hanging drop method at $18{ }^{\circ} \mathrm{C}$. Drops containing $1.5 \mu \mathrm{l}$ of purified $\mathrm{MDH}$ protein at $80 \mu \mathrm{M}$ containing $0.4 \mathrm{mM}$ 6DHNAD were mixed with equal volumes of a reservoir solution containing 0.1 M MES, pH 6.5, 21\% PEG 8,000. Large crystals $(400 \times 200 \times 50 \mu \mathrm{m})$ formed in 1-2 weeks[23]. For data collection, crystals were retreived with a nylon cryo-loop and washed with $0.1 \mathrm{M} \mathrm{MES}, \mathrm{pH} 6.5,21 \%$ PEG 8,000, $0.5 \mathrm{mg} / \mathrm{ml} \mathrm{MDH}$ supplemented with 
$20 \%(\mathrm{v} / \mathrm{v})$ glycerol as a cryoprotectant. The cryo-solution and crystal were cooled to form a glass by immersion in liquid nitrogen.

L-lactate dehydrogenase (LDH) from rabbit muscle was purchased from SigmaAldrich as an ammonium sulfate suspension. The suspension was diluted five-fold with $50 \mathrm{mM}$ HEPES, pH 7.5, $50 \mathrm{mM} \mathrm{NaCl}, 1 \mathrm{mM}$ DTT, desalted using a PD-10 column (GE Healthcare) into the same buffer, and concentrated to $410 \mu \mathrm{M}$. LDH crystal growth was carried out by the hanging drop method at $18{ }^{\circ} \mathrm{C}$. A volume of $1.5 \mu \mathrm{L}$ of LDH protein containing $1.2 \mathrm{mM}$ 6DHNAD was mixed with an equal volume of a reservoir solution containing $0.1 \mathrm{M}$ Tris buffer, $\mathrm{pH}$ 7.5, $0.1 \mathrm{M}$ sodium acetate, 15\% PEG 8,000. Large crystals $(400 \times 300 \times 100 \mu \mathrm{m})$ formed in 3-4 days. For data collection, crystals were soaked with $0.3 \mu \mathrm{L}$ of $9.3 \mathrm{mM}$ 6DHNAD for 40 minutes and washed with $0.1 \mathrm{M}$ Tris buffer, $\mathrm{pH} 7.5,0.05 \mathrm{M}$ sodium acetate, $14 \%$ PEG 8,000 supplemented with $25 \%(\mathrm{v} / \mathrm{v})$ PEG 400 as a cryoprotectant and flash cooled in liquid nitrogen. Data collection and model refinement statistics are listed in Table 1.

All MDH and LDH diffraction data were collected at the Stanford Synchrotron Radiation Laboratory (SSRL; Stanford, CA), beamline 9-2. MDH•6DHNAD diffraction data were collected $\left(0.2^{\circ}\right.$ oscillation images for a total of $\left.131^{\circ}\right)$ at a wavelength of $0.9795 \AA$ at $100 \mathrm{~K}$. The exposure time per frame was $2.83 \mathrm{~s}$ with $0 \%$ attenuation and a crystal to detector distance of $280.2 \mathrm{~mm}$. The data were indexed and scaled with XDS to $1.75 \AA$. The crystals were assigned to the space group $\mathrm{P} 2{ }_{1} 2_{1} 2_{1}$ with unit cell dimensions $\mathrm{a}=77.5$ $\AA, b=83.8 \AA, c=89.3 \AA$. Molecular replacement calculations for MDH were performed using PHASER in the PHENIX program suite [24, 25], using molecule A of 3HHP[26] as the 
search model with waters removed, yielding a clear solution with a log-likelihood gain of 9,078 and a TFZ score of 89.0. Model building and refinement were performed in iterative cycles using COOT[27] and phenix.refine. The MDH structure model includes residues 1-78, 90-311 in molecule A and 1-79, 90-311 in molecule B of 312 total residues. The model includes two 6DHNAD molecules, one in each NADH binding site, and 473 water molecules.

$\mathrm{LDH} \bullet 6 \mathrm{DHNAD}$ diffraction data $\left(0.2^{\circ}\right.$ oscillation images for a total of $\left.180^{\circ}\right)$ were collected with a wavelength of $0.9795 \AA$ at $100 \mathrm{~K}$. The exposure time per frame was $0.860 \mathrm{~s}$ with $0 \%$ attenuation and a crystal to detector distance of $285.3 \mathrm{~mm}$. The data were indexed and scaled with XDS to $1.86 \AA$. The crystals were assigned to the space group $\mathrm{P} 2{ }_{1}$ with unit cell dimensions $\mathrm{a}=63.6 \AA \mathrm{A}, \mathrm{b}=126.1 \AA \mathrm{A}, \mathrm{c}=84.2 \AA$, and $\beta=99.9^{\circ}$. Data collection and model refinement statistics are listed in Table 1. Molecular replacement calculations for LDH were performed using PHASER in the PHENIX program suite, using molecule A from $419 \mathrm{H}[28]$ as the search model with ligand and waters removed yielding a clear solution with a log-likelihood gain of 15,192 with a TFZ score of 86.9. Model building and refinement were performed using COOT and phenix.refine. The LDH structure model includes residues 1-13, 17-331 in molecule A; 2-98, 109-331 in molecule B; 2-13, 17-329 in molecule C; and 1-329 in molecule D of 331 total residues. The model also includes 4 6DHNAD molecules, one in each $\beta$-NADH-binding site. The 6DHNAD in molecule D was best modeled with two orientations of the adenine ring. There are 506 water molecules and 5 sulfates modelled. The sulfate ions were derived from the ammonium sulfate suspension in which the protein was purchased. 
Structural analysis. Protein structure figures were generated using PyMOL [29]. The atomic coordinates and structure factors have been deposited in the Protein Data Bank (Research Collaboratory for Structural Bioinformatics, Rutgers University, New Brunswick, NJ) as entries 5KKA for the MDH•6DHNAD structure, 5KKC for the $\mathrm{MDH} \bullet 6 \mathrm{DHNAD}$ structure and 5KRQ for the PpRen $\beta$-NADPH structure.

Single crystal spectrophotometry. In order to verify that the MDH•6DHNAD and LDH $\bullet$ DHNAD structures contained 6DHNAD and not a decomposition product of this relatively unstable molecule, absorption spectra where recorded from the same crystals of these complexes that were used for structural elucidation. Absorbance spectra (220$500 \mathrm{~nm}$ ) were recorded on SSRL beamline $9-2$ by measuring counts for dark (D) and reference $(R)$ scans adjacent to the crystal and then focusing the beam on the crystal or sample (S) to measure transmittance. Absorption spectra were then obtained according to equation 4.

Equation $4 \quad A=\log _{10}[(R-D) /(S-D)]$

A five-axis pico-motor stage was used to align the microspec objective lenses with each other and three larger stages were used to align the pair of objectives to the sample position. An approximately $50 \mu \mathrm{m}$ diameter circular beam was used. The system used a Hamamatsu light source with both deuterium and halogen lamps, UV solarization- 
resistant optical fibers, reflective Newport Schwardchild objectives, and an Ocean Optics QE65000 Spectrum Analyzer. 


\section{Results}

HPLC substrate analysis of 2-, 4- and 6DHNMN and 2-, 4- and 6DHNR. All three reduced isomers of $\mathrm{NR}$ and $\mathrm{NMN}$ were reacted with renalase to determine which were substrates. Control reactions were run in the absence of enzyme to define initial reference concentrations and account for losses due to decomposition. Analysis of the chromatographs obtained indicated that, much like the $\beta-N A D(P)$-derived substrates, only 2- and 6DHNR and 2- and 6DHNMN are consumed by renalase (Figure $2 \mathrm{~A}$ and C). The extinction coefficient spectrum for the dihydronicotinamide chromophore was determined for each reduced form using spectrophotometry in conjunction with a dioxygen electrode. This procedure yielded extinction coefficients of $\varepsilon_{338 \mathrm{~nm}}=7100 \pm 300$ $\mathrm{M}^{-1} \mathrm{~cm}^{-1}$ for 4DHNMN, $\varepsilon_{338 \mathrm{~nm}}=7300 \pm 200 \mathrm{M}^{-1} \mathrm{~cm}^{-1}$ for $6 \mathrm{DHNMN}, \varepsilon_{391 \mathrm{~nm}}=7000 \pm 100 \mathrm{M}^{-}$ ${ }^{1} \mathrm{~cm}^{-1}$ for 2 DHNMN, $\varepsilon_{340 \mathrm{~nm}}=7200 \pm 100 \mathrm{M}^{-1} \mathrm{~cm}^{-1}$ for 4DHNR, $\varepsilon_{345 \mathrm{~nm}}=10400 \pm 600 \mathrm{M}^{-1} \mathrm{~cm}^{-}$

${ }^{1}$ for 6 DHNR and $\varepsilon_{389 \mathrm{~nm}}=8700 \pm 500 \mathrm{M}^{-1} \mathrm{~cm}^{-1}$ for 2DHNR (Figure $2 \mathrm{~B}$ and D).

Reductive half-reactions and the effect of complimentary ligands with 2-and 6DHNR and 2- and $6 D H N M N$. We have previously reported that 2- and 6DHNAD(P) molecules are substrates for renalase. Here we report data for reductive half reactions of renalase from both $H$. sapiens and $P$. phaseolicola with 2- and 6DHNR and 2- and 6DHNMN as substrates (Figure 2) with and without the complimentary co-ligand of each: ADP or AMP, respectively (Figure 3). Similar to the $\beta$-NAD- and $\beta$-NADP-derived substrates, the $6 \mathrm{DH}$ isomers of NR and NMN proved sufficiently stable to be purified, desalted and stored as described above and the $2 \mathrm{DH}$ isomers were considerably less stable, dictating 
1 that they be used immediately after elution from preparative HPLC. As such the

2 concentration of the $2 \mathrm{DH}$ substrates was limited by the ability of the semi preparative

3 C18 column to resolve the $2 \mathrm{DH}$ isomer from other species present ( $100 \mu \mathrm{M}$ for 2DHNR

4 and 2DHNMN). Observation of the reduction of the flavin cofactor (458 $\mathrm{nm}$ for HsRen

5 and $450 \mathrm{~nm}$ for PpRen) by 2- and 6DHNR and 2 and $6 \mathrm{NMN}$ in the presence or absence of

6 the respective compliment ligand (ADP for nicotinamide riboside species or AMP for

7 nicotinamide mononucleotide) was carried out under anaerobic conditions at $25{ }^{\circ} \mathrm{C}$. The

8 complimentary ligand at high concentration (generally limited by solubility) was mixed

9 with renalase in the first mixing step and allowed to age for 100 ms. Reduction

10 experiments used largely pseudo-first order concentration ranges: 6DHNR (57 to 949

$11 \mu \mathrm{M}), 6 \mathrm{DHNMN}$ (78 to $675 \mu \mathrm{M}), 2 \mathrm{DHNR}$ (32 $\mu \mathrm{M}$ to $105 \mu \mathrm{M}$ ) and 2DHNMN (47 $\mu \mathrm{M}$ to 83

$12 \mu \mathrm{M})$. These data were fit to a single exponential decay to obtain a measure of observed

13 rate constant values (Equation 1$)$.

14 Plotting $k_{\text {obs }}$ data versus the concentration of substrate (2- and 6DHNR +/- ADP,

15 2- and 6DHNMN +/- AMP) gave a linear dependence for all data sets, where the slope is

$16 \mathrm{k}_{\mathrm{red}} / \mathrm{K}_{\mathrm{d}}$. A linear dependence suggests that the reduction reaction is reliant on relatively

17 weak binding interactions for these substrates, and the concentrations attained were

18 insufficient to show curvature in the dependence plot that would be indicative of pre-

19 equilibrium binding. For PpRen the addition of complementary or counterpart ligands

20 (AMP for NMN, ADP for NR) had no significant effect. For HsRen, addition of ADP (4-5

$21 \mathrm{mM}$ ) in together with 6DHNR resulted in a 7-fold increase in $\mathrm{k}_{\text {red }} / \mathrm{K}_{\mathrm{d}}$ (Figure 3 ), but the

22 equivalent analysis for 2- and 6DHNMN with HsRen with the AMP counterpart ligand 
1 showed no measurable difference for $\mathrm{k}_{\text {red }} / K_{\mathrm{d}}$. These data suggest that the $\beta$-phosphate

2 of bound ADP induces a conformation change that aids in hydride transfer from

$36 \mathrm{DHNMN}$ to the Flavin. More generally, however, the data show that untethered

4 occupancy of the distal portions of the dinucleotide binding site do not dramatically

5 enhance the rate of hydride transfer. For all substrates, maximal rates for hydride

6 transfer are observed with intact dinucleotides.

8 Inhibition of renalase by $\beta-N A D(P)$ analogs. Dissociation constants for non-substrate

9 fragments and isomers were measured by determination of the $50 \%$ suppression (I $\left.\mathrm{C}_{50}\right)$

10 of the observed rate constant for reduction in the presence of a substrate (6DHNAD)

11 concentration equal to the $K_{d}$ for that substrate (a concentration that was also pseudo-

12 first order with respect to the enzyme concentration). For those molecules that were

13 inhibitory, IC $C_{50}$ values were calculated by fitting a plot of $k_{\text {obs }}$ for reduction versus the

14 concentration of the substrate fragments, AMP, ADP, ADP-ribose, nicotinamide, methyl

15 nicotinamide, NR or NMN to equation 3 (Figure 4). This analysis was hampered to some

16 extent by apparent $\mathrm{IC}_{50}$ values greater than or similar to the solubility limit for these

17 molecules. However, the data show that it is generally the case that the phospho-group

18 proximal to the nicotinamide has a greater contribution to ligand binding than does the

19 distal. With the exception of $4 \mathrm{DHNR}$, molecules that did not contain this proximal

20 phospho group, such as AMP, nicotinamide and methyl nicotinamide, did not show

21 measureable inhibition of 6DHNAD oxidation. However, derivatives that did contain the

22 proximal phosphate, ADP, ADP-ribose and $4 D H N M N$, displayed observable inhibition 
1 yielding estimated $K_{d}$ values of $15,12.4$ and $1 \mathrm{mM}$, respectively. Titration of oxidized

2 forms of NR and NMN did not yield any substrate inhibition even though the 4-dihydro

3 form of both molecules was observed to be inhibitory. Moreover, the products ( $\beta-\mathrm{NAD}^{+}$

4 and $\beta$-NADP ${ }^{+}$) have been shown to bind with low millimolar binding constants. This likely

5 reflects competing cumulative interactions at the active site and along the ligand

6 binding cleft. The positive charge of the oxidized nicotinamide is likely repelled from the

7 active site by the local positive electrostatic environment adjacent to the nicotinamide

8 binding site resulting from conserved histidine and arginines (Figure 1), and we have

9 proposed in prior work that this potential aids product dissociation[14]. Truncated and

10 reduced non-substrate isomers (4DHNR, 4DHNMN) avoid this repulsive influence but

11 cannot form other interactions with the AMP/ADP moiety that would aid binding. We

12 therefore conclude that, in the oxidized state $\left(\mathrm{NR}^{+}\right.$and $\left.\mathrm{NMN}^{+}\right)$, the active site charge-

13 repulsion is dominant over other binding interactions.

15 The PpRen $\bullet$ NADP complex. Our prior investigation of renalase from $P$. phaseolicola

16 indicated that this form of renalase exhibited a pronounced substrate preference

17 profile. The bacterial enzyme reacted with $2 \mathrm{DH}$-substrates 160 to 350 -fold more rapidly

18 than 6DH-substrates. This bias seemed to be accounted for by a hydrogen bond from

19 the nicotinamide amide to threonine 185 that stabilized a substrate binding pose in

20 which the 2-dihydro hydride was proximal (3.6 $\AA$ ) and the nicotinamide ring parallel to

21 the flavin N-5 (Figure 1)[14]. As mentioned above, this form of renalase also bound $\beta$ -

22 NAD-derived substrates (6DH and $2 \mathrm{DH})$ and non-substrates (4DH) 15 to 50-fold more 
1 tightly than those derived from $\beta$-NADP, indicating that the added 2'-phosphoryl group

2 impedes binding by $6-10 \mathrm{~kJ} / \mathrm{mole}$. Such a substrate binding preference would be

3 expected if the function of renalase is to ameliorate the inhibitory threat to primary

4 metabolism where $\beta$-NAD is the predominant nicotinamide dinucleotide co-substrate.

5 The structural basis for this substrate preference is described here with the structure of

6 the PpRen $\beta$-NADPH complex, which was solved to $2.1 \AA$ A-resolution (Figure 5). This

7 structure is very similar to the PpRen $\beta$-NADH structure (4ZCC) we previously published,

8 however, the nicotinamide nucleoside moiety is disordered and not visible in the

9 electron density maps. The most striking aspect of this structure is that the NADPH 2'-

10 phosphoryl group does not appear to interact favorably or unfavorably, with regard to

11 binding, with any residue from the protein. While it is near the terminal amide of

12 glutamine 206 (3.4 $\AA$ ), the $\beta$-carbon of phenylalanine 204 (3.9 $\AA$ ) and aspartate $211(\sim 6.0$

$13 \AA$ A), none of these residues are oriented to form energetically significant binding

14 interactions. It therefore seems likely that for the bacterial enzyme $\beta$-NADP-derived

15 ligands are to a greater extent partitioned into the aqueous medium than those derived

16 from $\beta-N A D$ and that this is the basis for the NAD vs NADP ligand selectivity.

18 The $M D H \bullet 6 D H N A D$ and $L D H \bullet 6 D H N A D$ complexes. The final composite omit maps for

19 the $\mathrm{MDH} \bullet 6 \mathrm{DHNAD}$ or $\mathrm{LDH} \bullet 6 \mathrm{DHNAD}$ structures showed that two 6DHNAD inhibitor

20 complex structures were obtained. The MDH•6DHNAD structure was refined to $1.75 \AA$ -

21 resolution and the LDH•6DHNAD structure was refined to $1.86 \AA$-resolution (Figure 6).

22 These structures indicate, as might be predicted, that 6DHNAD occupies the NAD $(H)$ - 
1 binding site of both enzymes and does not appear to promote the binding of the

2 respective co-substrate. As a consequence of the high affinity of 6DHNAD for MDH and

$3 \mathrm{LDH}$, these structures serve as two examples of the inhibitory threat posed by non-

4 specific reduction of $\mathrm{NAD}^{+}$molecules.

5 There are four structures of $\mathrm{MDH}$ available on the PDB database; $\mathrm{MDH} \bullet \mathrm{NAD}^{+}$

6 (1IB6), $\mathrm{MDH} \bullet \mathrm{NAD}^{+} \bullet$ pyruvate $(1 \mathrm{IE} 3), \mathrm{MDH} \bullet \mathrm{NAD}^{+} \bullet$ citrate $(1 \mathrm{EMD})$, and a high resolution

7 structure of the unliganded enzyme (3HHP) [23, 30,31]. The binding of the 6DHNAD to

$8 \mathrm{MDH}$ is positionally identical to the other published $\mathrm{MDH} \bullet N A D$ structures with the

9 exception of $\mathrm{MDH} \bullet \mathrm{NAD}^{+} \bullet$ citrate (1EMD) where the nicotinamide ring is pivoted to

10 accommodate the citrate. While the omit map suggests a small pucker of the

11 nicotinamide ring at position 6, cyclohexadiene or piperidine-diene rings do not

12 normally have appreciable out of plane distortions. The overall structure of the MDH

13 molecules from all of the structures (those published and those presented here) are

14 very similar, with the only difference being in the loop (residues $81-90$ ) that closes

15 over the active site when citrate (or sulfate) is present (1EMD, 1IB6). This loop is open in

16 the $\mathrm{MDH} \bullet 6 \mathrm{DHNAD}$ structure presented here $(5 \mathrm{KKA})$, in the prior unliganded structure

17 (3HHP), and in the structure with pyruvate (1IE3). This loop is disordered in several of

18 the MDH molecules in the asymmetric units of these structures, whether there is

19 substrate bound or not.

20 There are also several structures of LDH in the PDB. There is a prior structure of

21 the rabbit muscle $\mathrm{LDH} \bullet \mathrm{NAD} \bullet$ oxamate ternary complex $(3 \mathrm{H} 3 \mathrm{~F})$ and 4 other structures

22 with a variety of inhibitors bound[32]. There is also a structure of the human muscle 
$1 \mathrm{LDH} \bullet N A D \bullet$ oxalate complex $(4 \mathrm{OKN})[33]$. The structure of rabbit muscle $\mathrm{LDH} \bullet 6 \mathrm{DHNAD}$

2 presented here aligns well with both the rabbit and human structures and the NAD

3 aligns well in all of the structures. The 6DHNAD in the LDH structure presented here

$4 \quad(5 \mathrm{KKC})$ also appears to have a small pucker in the nicotinamide ring. Similar to the MDH

5 structures, there is a loop that closes over the active site (residues 97-107) that is

6 disordered in all of the molecules of our structure's asymmetric unit, but is seen in the

7 open and closed positions in the other two structures, irrespective of whether there is a

8 substrate analog or inhibitor bound.

10 6DHNAD Crystal Occupancy from Single Crystal Spectrophotometry. 6DHNAD is a

11 relatively unstable molecule that degrades with a half-life of around 24 hours under the

12 conditions used for crystallization (data not shown). For both the MDH•6DHNAD and

$13 \mathrm{LDH} \bullet 6 \mathrm{DHNAD}$ structures, the crystals were formed by co-crystallization with the

14 inhibitory ligand. LDH was exposed to the ligand again prior to mounting in the loop

15 during cryo-protection. To verify occupancy of 6DHNAD in both the MDH and LDH

16 structures, single crystal spectrophotometry was performed on both complexes (Figure

17 6). For both $\mathrm{MDH}$ and $\mathrm{LDH}$, the the extinction coefficient of the GDHNAD nicotinamide

18 increases when bound. For the MDH $\bullet 6$ DHNAD complex the 6DHNAD $\lambda$ max shifts from

$19345 \mathrm{~nm}$ to $\sim 360 \mathrm{~nm}$, while for the LDH•6DHNAD complex the 6DHNAD

20 dihydronicotinamide $\lambda \max$ wavelength is largely unaltered when bound to the enzyme.

21 For the $\mathrm{MDH} \bullet 6 \mathrm{DHNAD}$ complex the extinction coefficient of the dihydronicotinamide

22 absorption transition increases by a factor of 1.14 , while for the LDH 6 DHNAD complex 
1 it increases by a factor of 1.23 . Both crystals exhibited far UV absorption features

2 indicative of a bound dihydronicotinamide establishing that 6DHNAD occupies the active

3 site of the crystals used to solve the MDH and LDH structures presented.

4 


\section{Discussion}

2 The data presented here and in prior work are consistent with an intracellular

3 housekeeping function for renalase. Non-specific reduction of $\mathrm{N}$-substituted

4 nicotinamides (chiefly $\mathrm{NAD}(\mathrm{P})^{+}$) yields three forms of the dihydronicotinamide base. We

5 have shown that two such isomers derived from reduction of $\mathrm{NAD}^{+}$are inhibitors of

6 enzymes that require $\mathrm{NADH}$ molecules as substrates. Renalase serves to oxidize these

7 inhibitory isomers and recycle them by forming $\mathrm{NAD}(\mathrm{P})^{+}($Scheme 1$)$. Prior work has

8 shown that renalases isolated from different kingdoms have the same activity but

9 unique structural features and substrate specificity profiles [13, 14]. In this study we

10 present data that accounts for some aspects of the specificity profile of human and

11 bacterial renalase, explore binding to the active site using analog and truncated

12 substrate and non-substrate molecules, and also offer structures of one inhibitory NADH

13 isomer (6DHNAD) bound to two primary metabolism dehydrogenases.

14 To date, only Human (HsRen) and Pseudomonas (PpRen) forms of renalase have

15 been identified. The structures of these enzymes indicate that the portion of the active

16 site of renalase that interacts with the FAD isoalloxazine and substrate/product

17 nicotinamide has few conserved residues. For both forms of the enzyme, the remainder

18 of the active site is an extended cleft on the surface of the protein. Ligand-bound

19 structures of PpRen indicate that this cleft accommodates the remainder of the

20 substrate and forms apparent hydrogen bonds and charge pairing interactions with the

21 phospho groups and the adenine base; moieties that are common to all mono- and

22 dinucleotide substrates and/or cofactors (Figure 1). Renalase functions in an 
1 intracellular environment in which potentially competing inhibitory ligands predominate

2 (AMP, ADP, ATP, NADH, NADPH, 4DHNR \& 4DHNMN) relative to the concentration of

3 available substrates (6DHNAD, 2DHNAD, 6DHNAP, 2DHNADP, 6DHNR, 2DHNR, 6DHNMN

4 and $2 \mathrm{DHNMN}$ ), that are assumed to be maintained at low concentration by the

5 irreversible oxidase activity of renalase. Our data indicate that non-substrate ligands

6 tend to either bind weakly (or possibly do not bind) to renalase (Figure 4). Nicotinamide

7 ribosides and nucleotides are predominantly found in four forms in the cell: NAD, NADP,

8 NR and NMN. We have shown that the 2- and 6-dihydronicotinamides of each of these

9 molecules are substrates for renalase [2] (Figure 2) and that renalase has the capacity to

10 kinetically select against oxidizing 4-dihydronicotinamide substrates by positioning the

11 nicotinamide such that the FAD N5, the presumed site for hydride transfer [34], is

12 distant from the 4-position of the nicotinamide [14]. The NR and NMN substrates bind

13 to renalase weakly (Figure 3 ), to the extent that no curvature was observed in the plot

14 of the observed rate constant for reduction versus substrate concentration, suggesting

15 quasi-collision based chemistry within the limited range of substrate concentration

16 available to assess these dependencies (Figure 3). However, these molecules behave as

17 substrates as they reduce the renalase cofactor approximately 5-orders of magnitude

18 more rapidly than does $\beta$-NADH (4DHNAD). If the substrate forms of NR and NMN

19 bound in the same conformation as that portion of $\beta-N A D(P)(H)$ observed in the

20 PpRen $\bullet \beta-N A D H$ and PpRen $\bullet \beta-N A D^{+}$structures[14], NR could make only one hydrogen

21 bond and NMN would form a maximum of two hydrogen bonds and two

22 complementary charge-pair interactions accounting for the six-fold difference in the $K_{d}$ 
1 for 4DHNMN compared to 4DHNR (Figure 1). One curiosity that was observed for HsRen

2 is that the simultaneous addition of a high concentration of the complement co-ligand

3 (ADP for NR) stimulated the rate of reduction by a factor of seven for 6DHNR. This

4 emphasizes that the substrate-binding sites in HsRen and PpRen are fundamentally

5 different and suggests that, for HsRen, catalysis can be enhanced by occupancy of the

6 distant portions of the substrate binding cleft relative to the site of hydride transfer.

7 Moreover, it suggests that for HsRen an intact pyrophosphate moiety increases the rate

8 of reduction.

9 The structure of the PpRen $\beta$-NADPH complex was solved to account for the

10 unique specificity profile of the bacterial enzyme. PpRen exhibits a $6-10 \mathrm{~kJ} / \mathrm{mol}$

11 preference to bind NAD-derived molecules compared to NADP-derived ligands [14]. The

12 structure of the PpRen $\beta$-NADPH complex indicates that the 2'-phospho-group of $\beta$ -

13 NADPH does not interact in a manner conducive to binding with any residue on the

14 surface of the enzyme. As such, there is no partner residue to satisfy the negative

15 charge of the phospho group, dictating that this charged moiety would retain its

16 hydration shell when bound to PpRen. Such a binding pose would mean that $\beta$-NADPH

17 would be fractionally more partitioned into bulk solvent when bound, undermining its

18 capacity to remain associated. Comparing HsRen and PpRen, $\beta$-NAD and NADP-derived

19 ligands bind with similar affinity to HsRen [2], whereas PpRen binds NAD-derived ligands

20 with dissociation constants that indicate 13 to 50 -fold higher affinity than for ligands

21 derived from NADP [14]. This difference in dinucleotide specificity is potentially

22 accounted for by the presence of an arginine (Arg222) in HsRen whose guanidino group 
1 is adjacent the position occupied by the 2'-phospho group (as observed in the PpRen• $\beta$ -

2 NADPH structure; Figure 1). In the equivalent position of the PpRen $\beta$-NADPH structure

3 is an aspartate residue (Asp211) whose carboxylate resides $6.0 \AA$ from the $\beta$-NADPH 2'-

4 phospho group.

5 In prior studies, we demonstrated that 2- and 6DHNAD molecules were

6 competitively inhibitory to specific enzymes that have $\beta$-NADH as substrate [2]. The

7 assumption was that the isomeric forms are sufficiently similar in shape and charge

8 distribution to mimic $\beta$-NADH molecules. These isomers bound with $\mathrm{K}_{\mathrm{i}}$ values in the sub-

9 micromolar range with E.coli malate dehydrogenase exhibiting a $K_{i}$ of $34 \mathrm{nM}$ for

10 6DHNAD. Here we present the structures of E.coli malate dehydrogenase and rabbit

11 muscle lactate dehydrogenase bound to 6DHNAD. Instability of the 2- and 6DHNAD

12 molecules dictates that we verify that these molecules are present in the structures as

13 solved. To do this we used the dihydro-nicotinamide chromophore to observe the

14 absorption of the ligand by single crystal spectrophotomtery (Figure 6). The spectra

15 obtained indicate that both the MDH and LDH structures were solved with 6DHNAD

16 bound (given that this was the only reduced form of NAD added). As may be readily

17 predicted, 6DHNAD occupies the binding site for $\beta-\mathrm{NADH} / \beta-\mathrm{NAD}{ }^{+}$in these enzymes [31,

18 32], convincingly demonstrating two examples of the mode of competitive inhibition of

19 primary metabolism enzymes by 6DHNAD and by extension 2DHNAD molecules. 


\section{References}

3 [1] S.E. Godtfredsen, M. Ottesen, 1,6-Dihydro-NAD as an Humidity-Induced Lactate

4 Dehydrogenase Inhibitor in NADH Preparations, Carlesberg Research Communications $543(1978) 171-175$.

6 [2] B.A. Beaupre, M.R. Hoag, J. Roman, F.H. Forsterling, G.R. Moran, Metabolic Function

7 for Human Renalase: Oxidation of Isomeric Forms of beta-NAD(P)H that Are Inhibitory

8 to Primary Metabolism, Biochemistry 54(3) (2015) 795-806.

9 [3] F.C. Luft, Renalase, a catecholamine-metabolizing hormone from the kidney, Cell

10 Metab 1(6) (2005) 358-60.

11 [4] J. Xu, G. Li, P. Wang, H. Velazquez, X. Yao, Y. Li, Y. Wu, A. Peixoto, S. Crowley, G.V.

12 Desir, Renalase is a novel, soluble monoamine oxidase that regulates cardiac function

13 and blood pressure, The Journal of clinical investigation 115(5) (2005) 1275-80.

14 [5] R.R. Gosh, T.W.B. Gehr, D.A. Sica, S. Masilamani, I. Fakhry, R. Wang, E. Mcquire, S.

15 Gosh, Effect of renalase inhibition on blood pressure. , J Am Soc Nephrol 17 (2006) 208A

16 (abstract).

17 [6] G.V. Desir, Renalase deficiency in chronic kidney disease, and its contribution to

18 hypertension and cardiovascular disease, Curr Opin Nephrol Hypertens 17(2) (2008)

$19 \quad 181-5$

20 [7] G. Li, J. Xu, P. Wang, H. Velazquez, Y. Li, Y. Wu, G.V. Desir, Catecholamines regulate

21 the activity, secretion, and synthesis of renalase, Circulation 117(10) (2008) 1277-82. 
1 [8] X. Guo, L. Wang, H. Velazquez, R. Safirstein, G.V. Desir, Renalase: its role as a

2 cytokine, and an update on its association with type 1 diabetes and ischemic stroke,

3 Curr Opin Nephrol Hypertens 23(5) (2014) 513-8.

4 [9] L. Wang, H. Velazquez, J. Chang, R. Safirstein, G.V. Desir, Identification of a receptor

5 for extracellular renalase, PLoS One 10(4) (2015) e0122932.

6 [10] X. Guo, L. Hollander, D. MacPherson, L. Wang, H. Velazquez, J. Chang, R. Safirstein,

7 C. Cha, F. Gorelick, G.V. Desir, Inhibition of renalase expression and signaling has

8 antitumor activity in pancreatic cancer, Sci Rep 6 (2016) 22996.

9 [11] L. Hollander, X. Guo, H. Velazquez, J. Chang, R. Safirstein, H.M. Kluger, C. Cha, G.

10 Desir, Renalase expression by melanoma and tumor associated-macrophages promotes

11 tumor growth through a STAT3-mediated mechanism, Cancer Res (2016).

12 [12] G.V. Desir, Renalase is a novel renal hormone that regulates cardiovascular

13 function, J Am Soc Hypertens 1(2) (2007) 99-103.

14 [13] M. Milani, F. Ciriello, S. Baroni, V. Pandini, G. Canevari, M. Bolognesi, A. Aliverti,

15 FAD-binding site and NADP reactivity in human renalase: a new enzyme involved in

16 blood pressure regulation, Journal of molecular biology 411(2) (2011) 463-73.

17 [14] M.R. Hoag, J. Roman, B.A. Beaupre, N.R. Silvaggi, G.R. Moran, Bacterial Renalase:

18 Structure and Kinetics of an Enzyme with 2- and 6-Dihydro-beta-NAD(P) Oxidase Activity

19 from Pseudomonas phaseolicola, Biochemistry 54(24) (2015) 3791-802.

20 [15] B.A. Beaupre, B.R. Carmichael, M.R. Hoag, D.D. Shah, G.R. Moran, Renalase Is an

21 alpha-NAD(P)H Oxidase/Anomerase (JACS Spotlight Article), Journal of the American

22 Chemical Society 135(37) (2013) 13980-7. 
1 [16] Z. Otwinowski, W. Minor, Processing of X-ray diffraction data collection in

2 oscillation mode., Methods Enzymol 276 (1997) 307-325.

3 [17] A.J. McCoy, R.W. Grosse-Kunstleve, P.D. Adams, M.D. Winn, L.C. Storoni, R.J. Read,

4 Phaser crystallographic software, J Appl Crystallogr 40(Pt 4) (2007) 658-674.

5 [18] P. Emsley, B. Lohkamp, W.G. Scott, K. Cowtan, Features and development of Coot,

6 Acta Crystallogr D Biol Crystallogr 66(Pt 4) (2010) 486-501.

7 [19] P.V. Afonine, M. Mustyakimov, R.W. Grosse-Kunstleve, N.W. Moriarty, P. Langan,

8 P.D. Adams, Joint X-ray and neutron refinement with phenix.refine, Acta Crystallogr D

$9 \quad$ Biol Crystallogr 66(Pt 11) (2010) 1153-63.

10 [20] J.M. Word, S.C. Lovell, J.S. Richardson, D.C. Richardson, Asparagine and glutamine:

11 using hydrogen atom contacts in the choice of side-chain amide orientation, J Mol Biol

12 285(4) (1999) 1735-47.

13 [21] L. Urzhumtseva, P.V. Afonine, P.D. Adams, A. Urzhumtsev, Crystallographic model

14 quality at a glance, Acta Crystallogr D 65 (2009) 297-300.

15 [22] V.B. Chen, W.B. Arendall, 3rd, J.J. Headd, D.A. Keedy, R.M. Immormino, G.J. Kapral,

16 L.W. Murray, J.S. Richardson, D.C. Richardson, MolProbity: all-atom structure validation

17 for macromolecular crystallography, Acta Crystallogr D Biol Crystallogr 66(Pt 1) (2010)

$18 \quad 12-21$.

19 [23] J. Zaitseva, K.M. Meneely, A.L. Lamb, Structure of Escherichia coli malate

20 dehydrogenase at 1.45 A resolution, Acta crystallographica 65(Pt 9) (2009) 866-9.

21 [24] P.D. Adams, P.V. Afonine, G. Bunkoczi, V.B. Chen, I.W. Davis, N. Echols, J.J. Headd,

22 L.W. Hung, G.J. Kapral, R.W. Grosse-Kunstleve, A.J. McCoy, N.W. Moriarty, R. Oeffner, 
1 R.J. Read, D.C. Richardson, J.S. Richardson, T.C. Terwilliger, P.H. Zwart, PHENIX: a

2 comprehensive Python-based system for macromolecular structure solution, Acta

3 crystallographica. Section D, Biological crystallography D66(Pt 2) (2010) 213-21.

4 [25] P.V. Afonine, R.W. Grosse-Kunstleve, N. Echols, J.J. Headd, N.W. Moriarty, M.

5 Mustyakimov, T.C. Terwilliger, A. Urzhumtsev, P.H. Zwart, P.D. Adams, Towards

6 automated crystallographic structure refinement with phenix.refine, Acta

7 crystallographica 68(Pt 4) (2012) 352-67.

8 [26] J. Zaitseva, K.M. Meneely, A.L. Lamb, Structure of Escherichia coli malate

9 dehydrogenase at 1.45 A resolution, Acta Crystallogr Sect F 65(Pt 9) (2009) 866-9.

10 [27] P. Emsley, K. Cowtan, Coot: model-building tools for molecular graphics, Acta Cryst.

11 D60(Pt 12 Pt 1) (2004) 2126-32.

12 [28] A. Kohlmann, S.G. Zech, F. Li, T. Zhou, R.M. Squillace, L. Commodore, M.T.

13 Greenfield, X. Lu, D.P. Miller, W.S. Huang, J. Qi, R.M. Thomas, Y. Wang, S. Zhang, R.

14 Dodd, S. Liu, R. Xu, Y. Xu, J.J. Miret, V. Rivera, T. Clackson, W.C. Shakespeare, X. Zhu, D.C.

15 Dalgarno, Fragment growing and linking lead to novel nanomolar lactate dehydrogenase

16 inhibitors, Journal of medicinal chemistry 56(3) (2013) 1023-40.

17 [29] W. DeLano, The PyMOL Molecular Graphics System, http://www.pymol.org/,

18 DeLano Scientific, San Carlos, CA, 2002.

19 [30] J.K. Bell, H.P. Yennawar, S.K. Wright, J.R. Thompson, R.E. Viola, L.J. Banaszak,

20 Structural analyses of a malate dehydrogenase with a variable active site, J Biol Chem

$21 \quad 276(33)(2001) 31156-62$. 
1 [31] M.D. Hall, L.J. Banaszak, Crystal structure of a ternary complex of Escherichia coli

2 malate dehydrogenase citrate and NAD at 1.9 A resolution, J Mol Biol 232(1) (1993) 213-

322.

4 [32] K. Swiderek, A. Panczakiewicz, A. Bujacz, G. Bujacz, P. Paneth, Modeling of isotope

5 effects on binding oxamate to lactic dehydrogenase, J Phys Chem B 113(38) (2009)

$6 \quad 12782-9$.

7 [33] S. Kolappan, D.L. Shen, R. Mosi, J. Sun, E.J. McEachern, D.J. Vocadlo, L. Craig,

8 Structures of lactate dehydrogenase A (LDHA) in apo, ternary and inhibitor-bound

9 forms, Acta Crystallogr D Biol Crystallogr 71(Pt 2) (2015) 185-95.

10 [34] E.F. Pai, G.E. Schulz, The catalytic mechanism of glutathione reductase as derived

11 from x-ray diffraction analyses of reaction intermediates, J Biol Chem 258(3) (1983)

$12 \quad 1752-7$.

13 [35] A. Cortes, M. Cascante, M.L. Cardenas, A. Cornish-Bowden, Relationships between

14 inhibition constants, inhibitor concentrations for $50 \%$ inhibition and types of inhibition:

15 new ways of analysing data, The Biochemical journal 357(Pt 1) (2001) 263-8.

16 
1 Schemes

$2 \quad$ Scheme 1

3

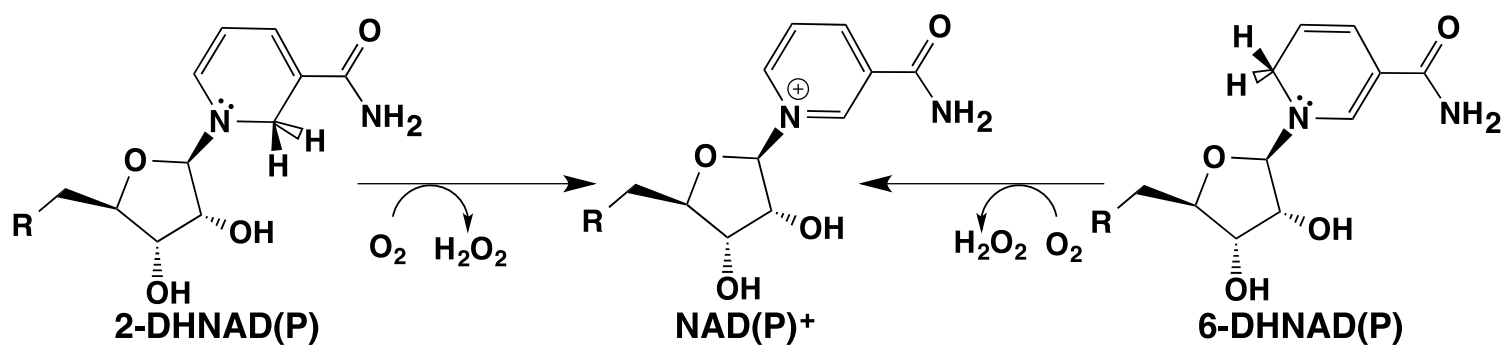

Nicotinamide mononucleotide (NMN) $\mathrm{R}=\mathrm{PO}_{4}{ }^{2-}$

$4 \quad$ Nicotinamide riboside (NR) $\mathrm{R}=\mathbf{O H}$ 


\section{Tables}

2 Table 1: Structural Statistics for the MDH•6DHNAD, LDH•6DHNAD and PpRen•NADPH

\section{3 complexes}

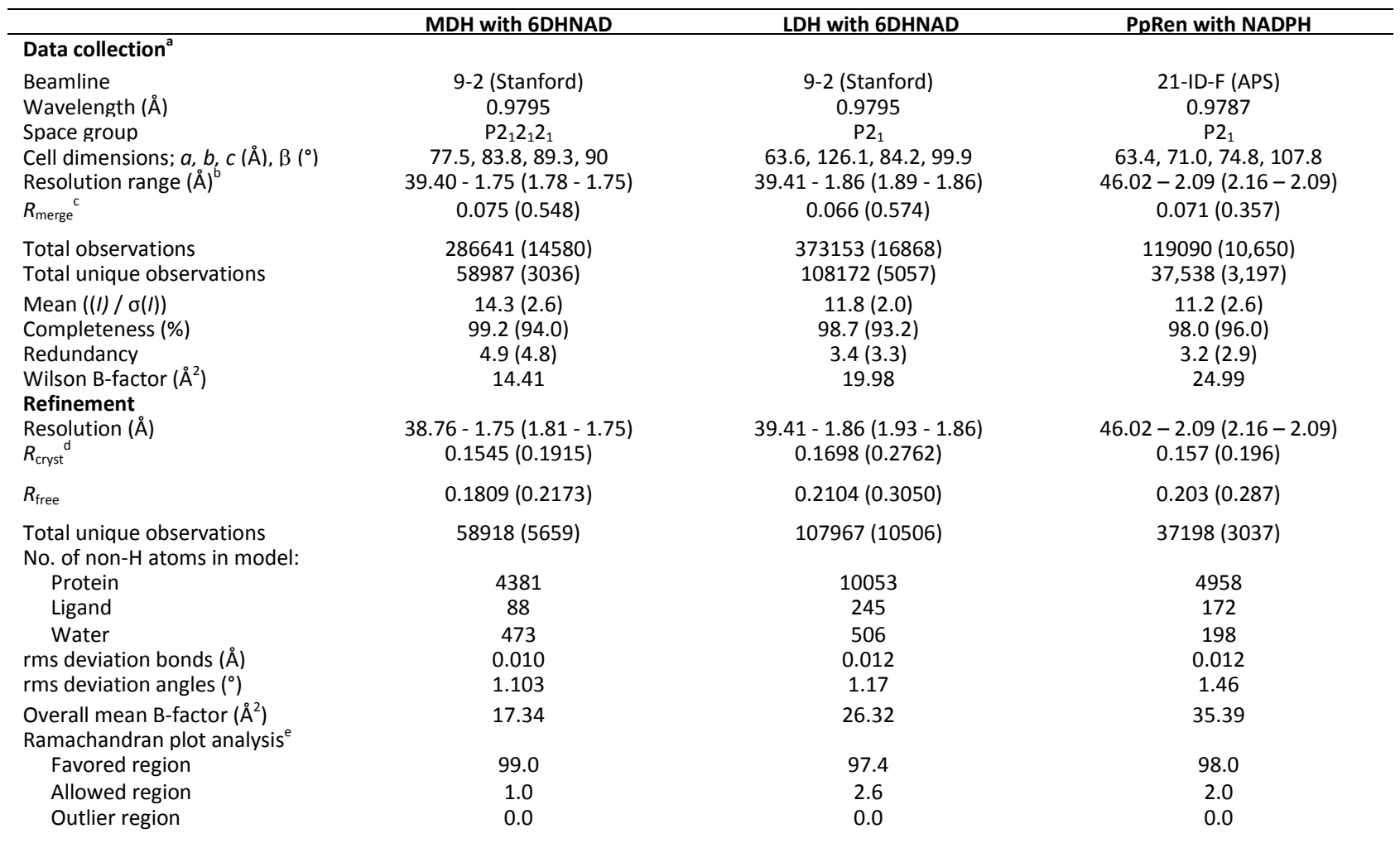

$4 \quad$ data indexed and scaled with XDS

5 values in paretheses are for the highest resolution shell

$6{ }^{c} R_{\text {merge }}=\Sigma_{h} \mid I_{h}-\left\langle|>| / \Sigma_{h} I_{h}\right.$, where $I_{h}$ is the intensity of reflection $h$, and $\langle>\rangle$ is the mean intensity of all symmetry-related 7 reflections

$8{ }^{\mathrm{d}} R_{c r y s t}=\Sigma|| F_{o}|-| F_{c}|| / \Sigma\left|F_{o}\right|, F_{o}$ and $F_{c}$ are observed and calculated structure factor amplitudes. Five percent of the

9 reflections were reserved for the calculation of $R_{\text {free }}$.

$10{ }^{d}$ Calculated with Molprobity 


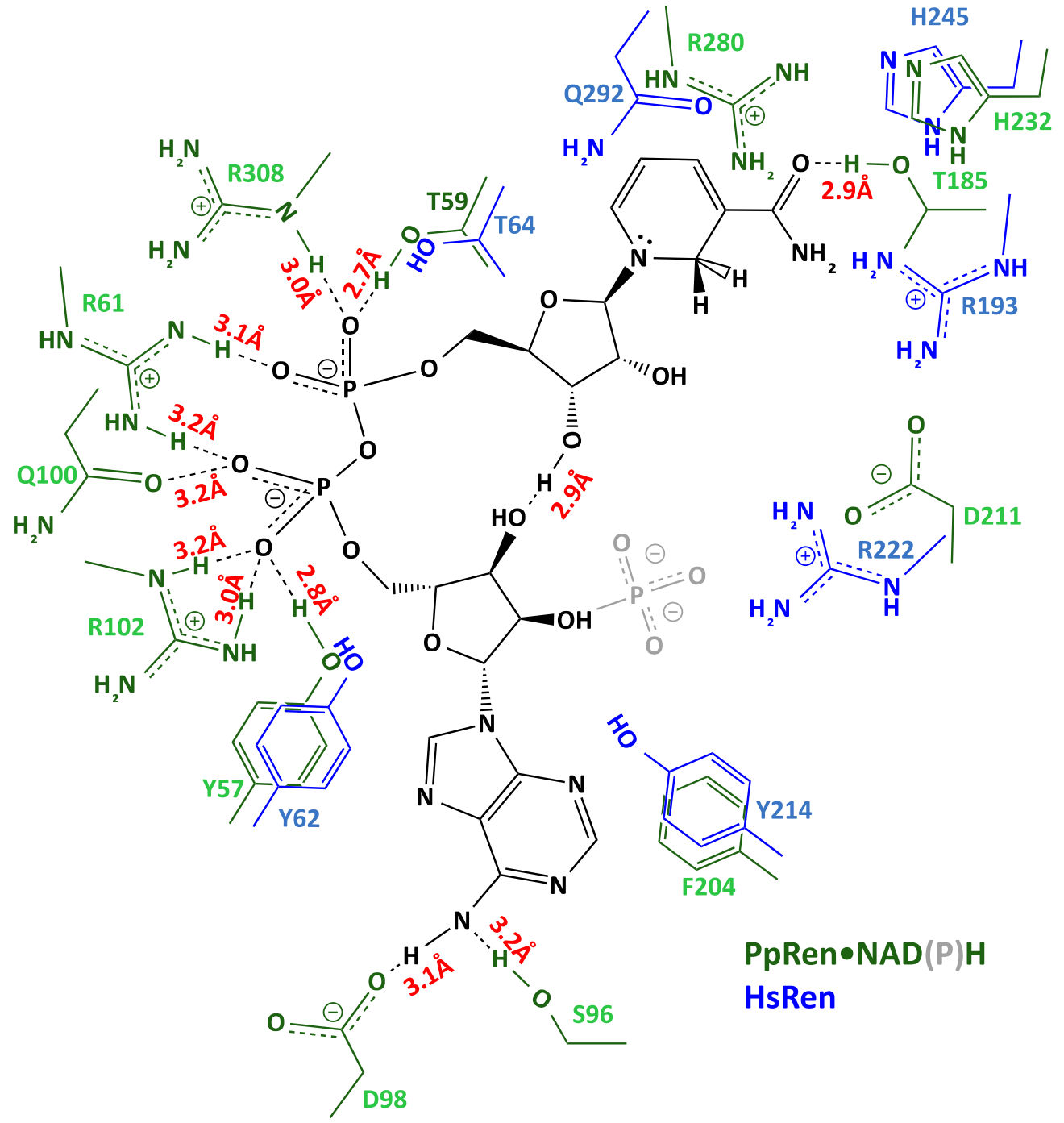

2 Figure 1: Two-Dimensional Representation of Active Site Interactions of NAD(P)H

3 Molecules with Bacterial and Human Renalase. Structure in black is the NAD(P)H

4 ligand; green indicates residues from Pseudomonas phaseolicola. Residues shown in

5 blue are the conserved and non-conserved residues from human renalase that are

6 proximal to the interacting residues from the $P$. phaseolicola structures. The 2'-

7 phosphoryl of NADPH is shown in grey. Apparent hydrogen bond and charge interaction

8 distances are shown in red 

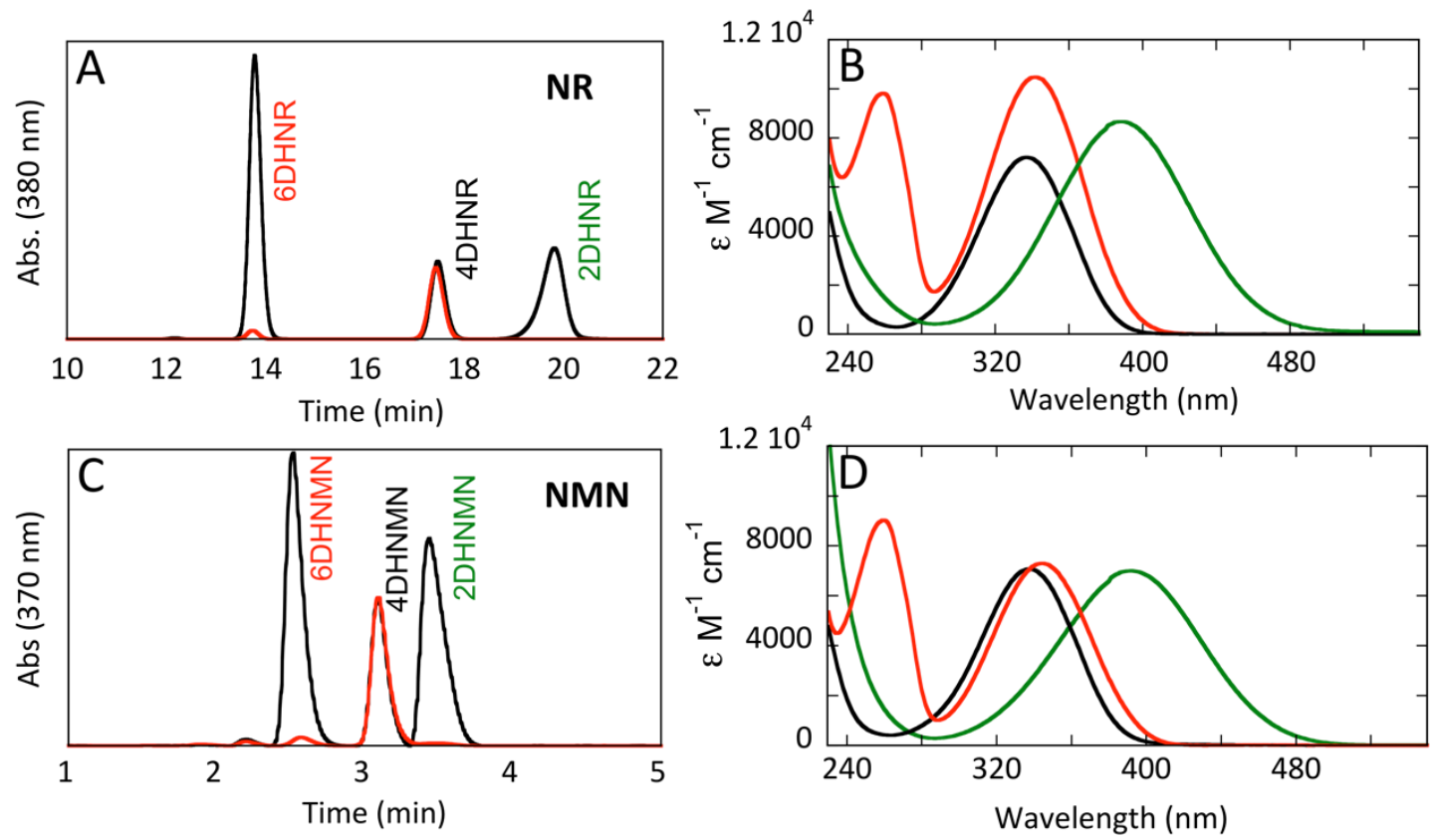

2 Figure 2. Oxidation of Dihydronicotinamide Nucleosides (NR) and Mononucleotide

3 (NMN) Substrates by Renalase. $\mathrm{NR}^{+}$and $\mathrm{NMN}^{+}$were reduced by the addition of sodium

4 borohydride forming three reduced isomers of each. These mixtures were then reacted

5 with renalase $(12.5 \mu \mathrm{M})$ for 10 minutes. A and C are HPLC chromatograms that showed

6 the three species formed by reduction without (black trace) and with (red trace) the

7 incubation with renalase. B and D are the extinction coefficient spectra for each of the

8 reduced forms obtained, each corresponding to the chromatograms shown to the left. 

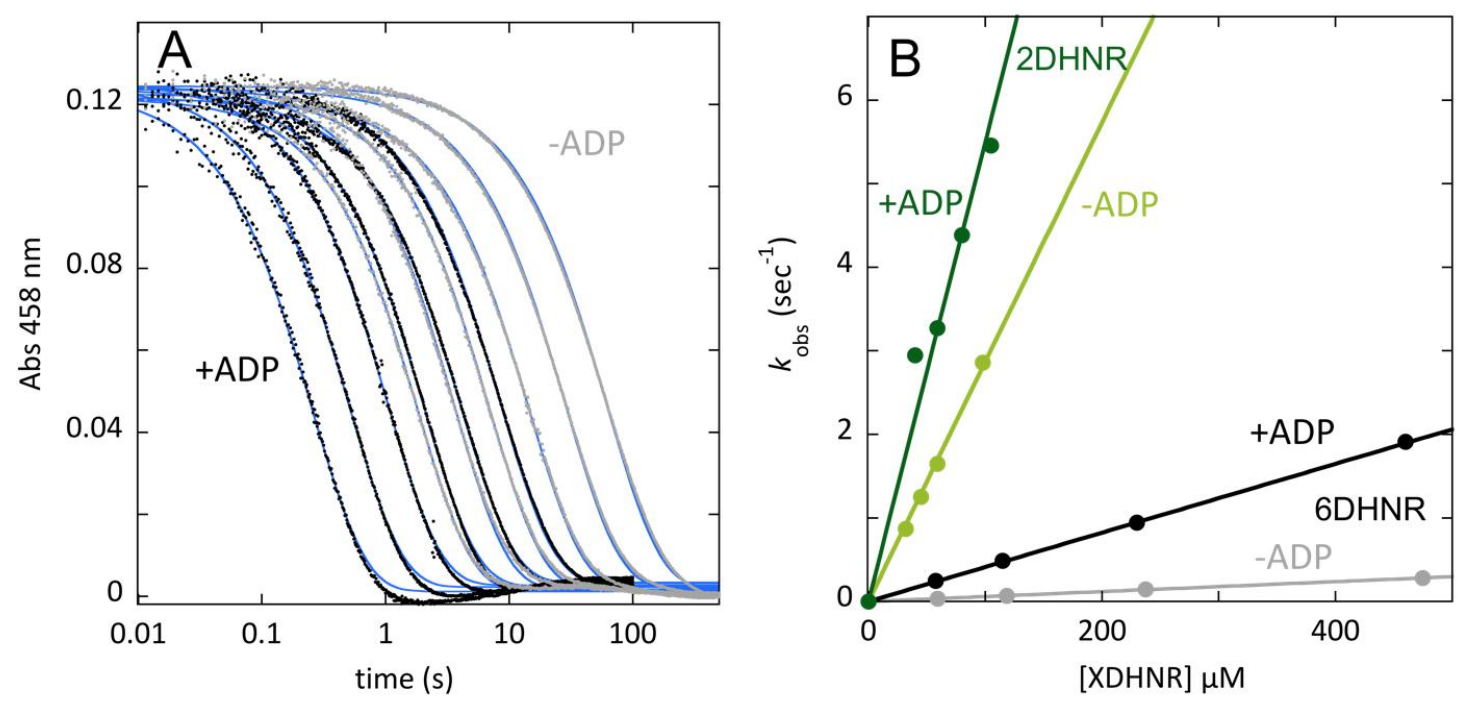

1

\begin{tabular}{llllll}
\hline Substrate & $\begin{array}{l}\text { Complimentary } \\
\text { Ligand }\end{array}$ & $\begin{array}{l}\mathbf{k}_{\text {red }} / \mathbf{K}_{\mathbf{d}} \mathbf{n M}^{-1} / \mathbf{s}^{-1} \\
\text { HsRen }\end{array}$ & Factor & $\begin{array}{l}\mathbf{k}_{\text {red }} / \mathbf{K}_{\mathbf{d}} \mathbf{n M}^{-1} / \mathbf{s}^{-1} \\
\text { PpRen }\end{array}$ & Factor \\
\hline 6DHNMN & +AMP (1.12 mM) & $30.0 \pm 0.3$ & & $0.032 \pm 0$ & \\
& -AMP & $30 \pm 1$ & 1.0 & $0.063 \pm 0.002$ & 2.0 \\
2DHNMN & AMP (1.12 mM) & $160 \pm 2$ & & $22 \pm 1$ & \\
& -AMP & $170 \pm 4$ & 1.1 & $26 \pm 2$ & 1.2 \\
6DHNR & ADP (3.95 mM) & $59 \pm 0$ & $4.1 \pm 0$ & $0.039 \pm 0.005$ & \\
& -ADP & 7.0 & $0.028 \pm 0.004$ & 0.7 \\
2DHNR & ADP (4.83 mM) & $29.0 \pm 0.3$ & & $1.0 \pm 0.1$ & \\
& -ADP & $55 \pm 3$ & 1.9 & $0.55 \pm 0.04$ & 0.6 \\
\hline
\end{tabular}

2 Figure 3. Reductive Half Reactions with 2- and 6Dihydro- NMN and NR with and

3 without complementary ligands. A. Representative reduction traces for HsRen (10 $\mu \mathrm{M})$

4 reacting with 6 DHNR in the presence (black) and absence (grey) of $4 \mathrm{mM} \mathrm{ADP}$. Data

5 were fit to a single exponential according to equation 1. Lines of best fit over the data

6 (blue). B. Secondary plot showing the effect of added ADP on the observed rate

7 constant for reduction of HsRen by both 2- and 6DHNAD. Data were fit to equation 2 to

8 determine values for $\mathrm{K}_{\text {red }} / \mathrm{K}_{\mathrm{d}}$ from the slope term. 


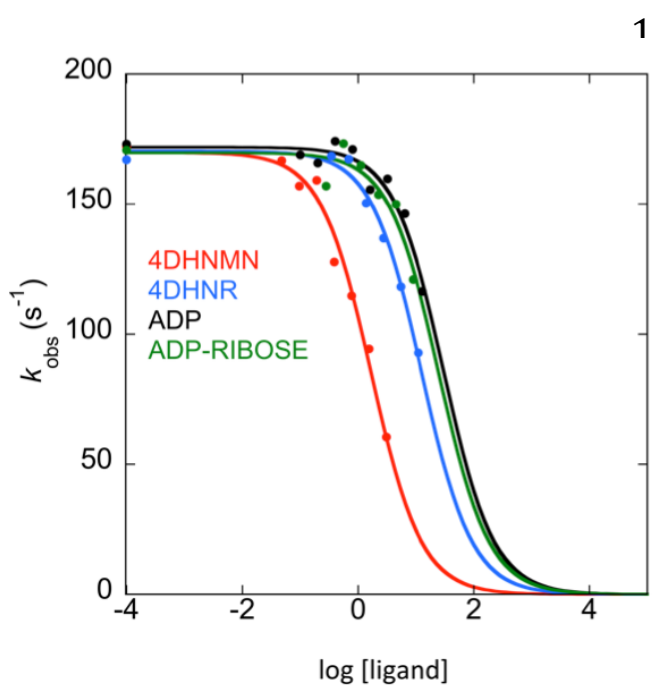

3

\begin{tabular}{ll}
\hline Ligand & $\mathbf{K}_{\mathbf{d}}(\mathbf{m M})$ \\
\hline ADP & $15.0 \pm 0.6^{a}$ \\
ADP-ribose & $12.4 \pm 0.5^{a}$ \\
NAD $^{+}$ & $2.8 \pm 0.3^{a}$ \\
4DHNR & $6.1 \pm 0.5^{a}$ \\
4DHNMN & $1.0 \pm 0.5^{a}$ \\
4DHNADP (NADPH) & $1.5 \pm 0.4^{b}$ \\
4DHNAD (NADH) & $0.6 \pm 0.1^{b}$ \\
6DHNAD & $0.17 \pm 0.01^{b}$ \\
2DHNAD & $0.17 \pm 0.01^{b}$ \\
\hline
\end{tabular}

$a$ - Values estimated from suppression
8 Figure 4. Estimates of Binding constants for substrate fragments and substrate analogs

9 with HsRen. The plot shows the partial $I C_{50}$ curves used to estimate the binding

10 constants of non-substrate ligands that exhibited inhibition. The fit of the curve to

11 equation 3 gave $I C_{50}$ values that by the methods used is twice the $K_{d}\left(\right.$ or $K_{i}$ ) for the

12 inhibitory ligand [35]. No evidence of inhibition of the reductive half reaction was

13 observed for AMP (7.6 mM), nicotinamide (68 mM), N-methyl-nicotinamide (7.8 mM),

$14 \mathrm{NR}^{+}$(7.3) $\left.\mathrm{mM}\right), \mathrm{NMN}^{+}(2.6 \mathrm{mM})$ with values in parentheses indicating maximal

15 concentration tested for evidence of inhibition. 


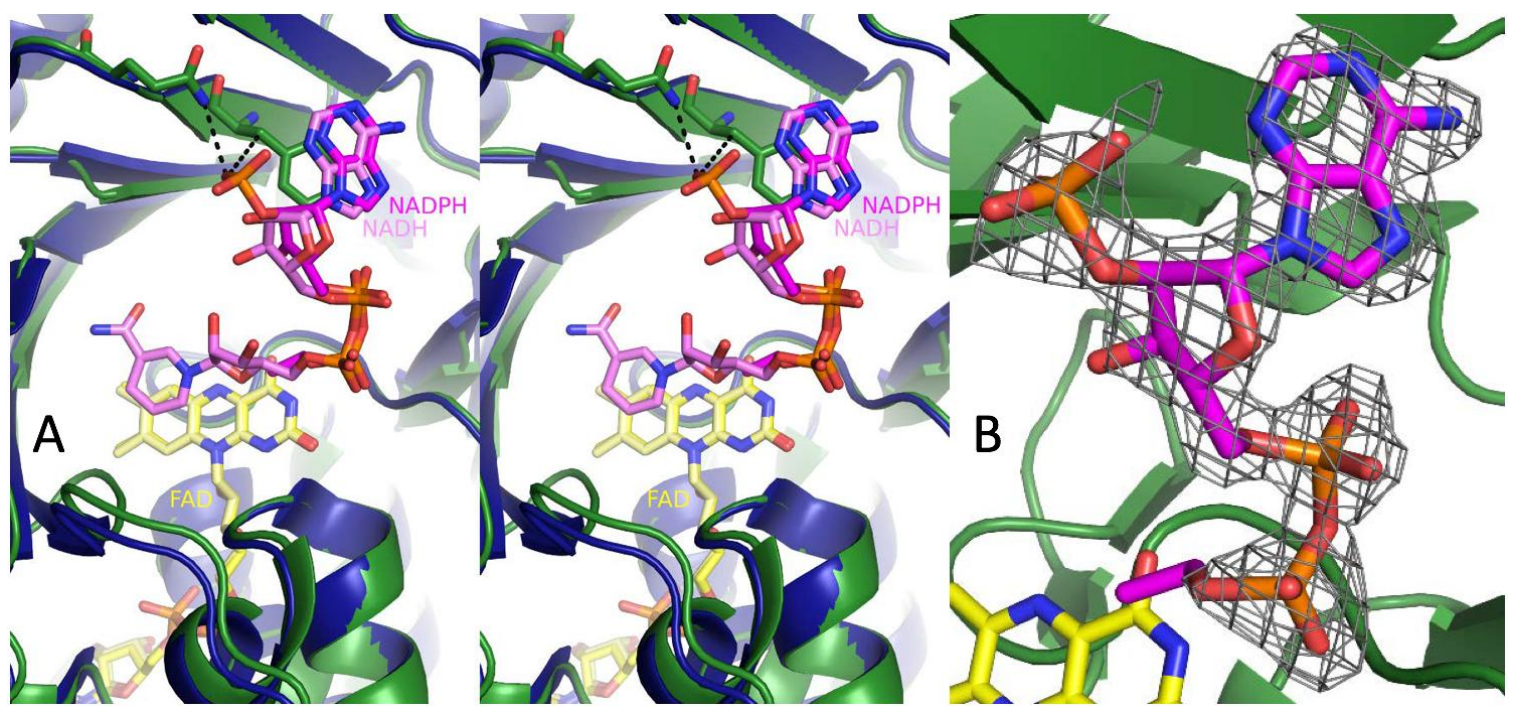

2 Figure 5. $\beta$-NADH vs $\beta$-NADPH Binding to PpRen A. Stereo view of the $\beta$-NADH-renalase

3 (PDB: 4ZCC, blue cartoon) overlaid on the NADPH-renalase (structure determined here,

4 green cartoon). FAD is colored with carbon atoms yellow for both molecules. The $\beta$-NADH is

5 shown with pink carbon atoms and the NADPH is magenta. The two most proximal residues

6 are shown with carbon atoms green. The phe204 ring stacks with the adenine ring of $\beta$ -

$7 \mathrm{NAD}(\mathrm{P}) \mathrm{H}$. The $\beta$-carbon of phe204 is $3.9 \AA$ from one oxygen of the 2-phosphoryl group. The

8 amide-nitrogen of gln206 is $3.4 \AA$ from the same oxygen; however, the density is poor for

9 this sidechain. Note that the FAD is buried, and that the $\beta-N A D(P) H$ is bound to a cleft on

10 the exterior of the protein. B. A simulated annealing omit map contoured at $3 \sigma$ surrounds

11 the $\beta$-NADPH (omitted in calculation) shows that the nicotinamide and nicotinamide-ribose

12 of NADPH are not resolved in the map. 


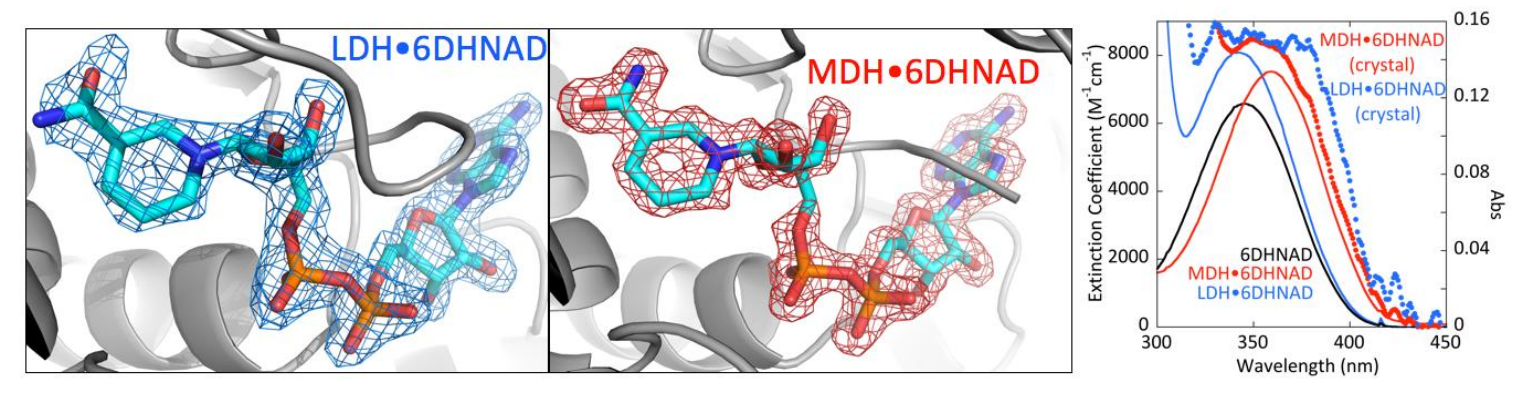

2 Figure 6. 6DHNAD inhibitory complex structures with MDH and LDH with 6DHNAD

3 bound. Structural figures show that simulated annealing omit maps contoured at $3 \sigma$

4 surround the 6DHNAD. C. Absorption Spectra recorded in solution (solid lines) and from the

5 crystals used to solve the structures shown at left (dotted lines). These spectra show the

6 presence of the 6DHNAD dihydronicotinamide chromophore in the crystals used to solve

7 the structures. 
Graphical Abstract

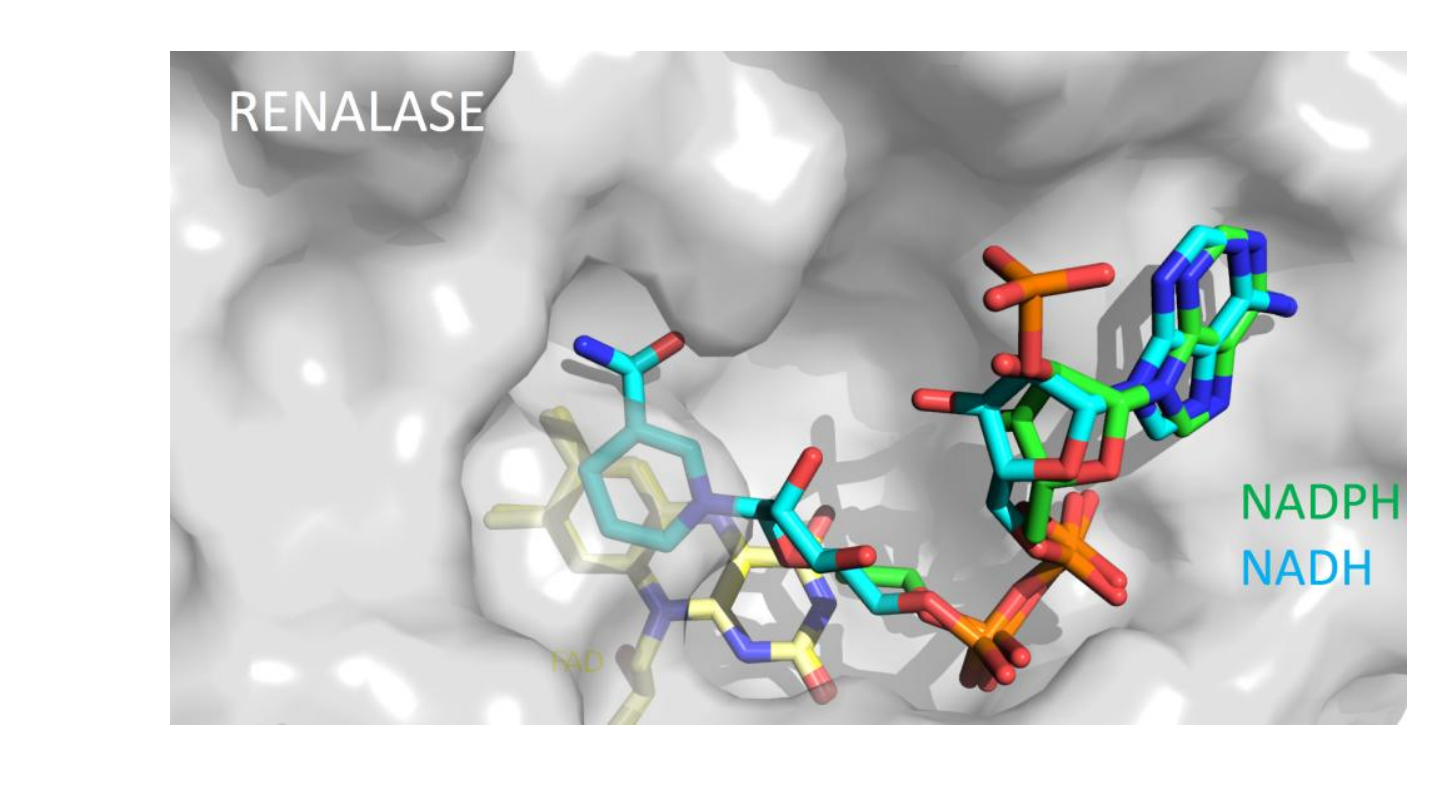 \\ Graphical Abstract}
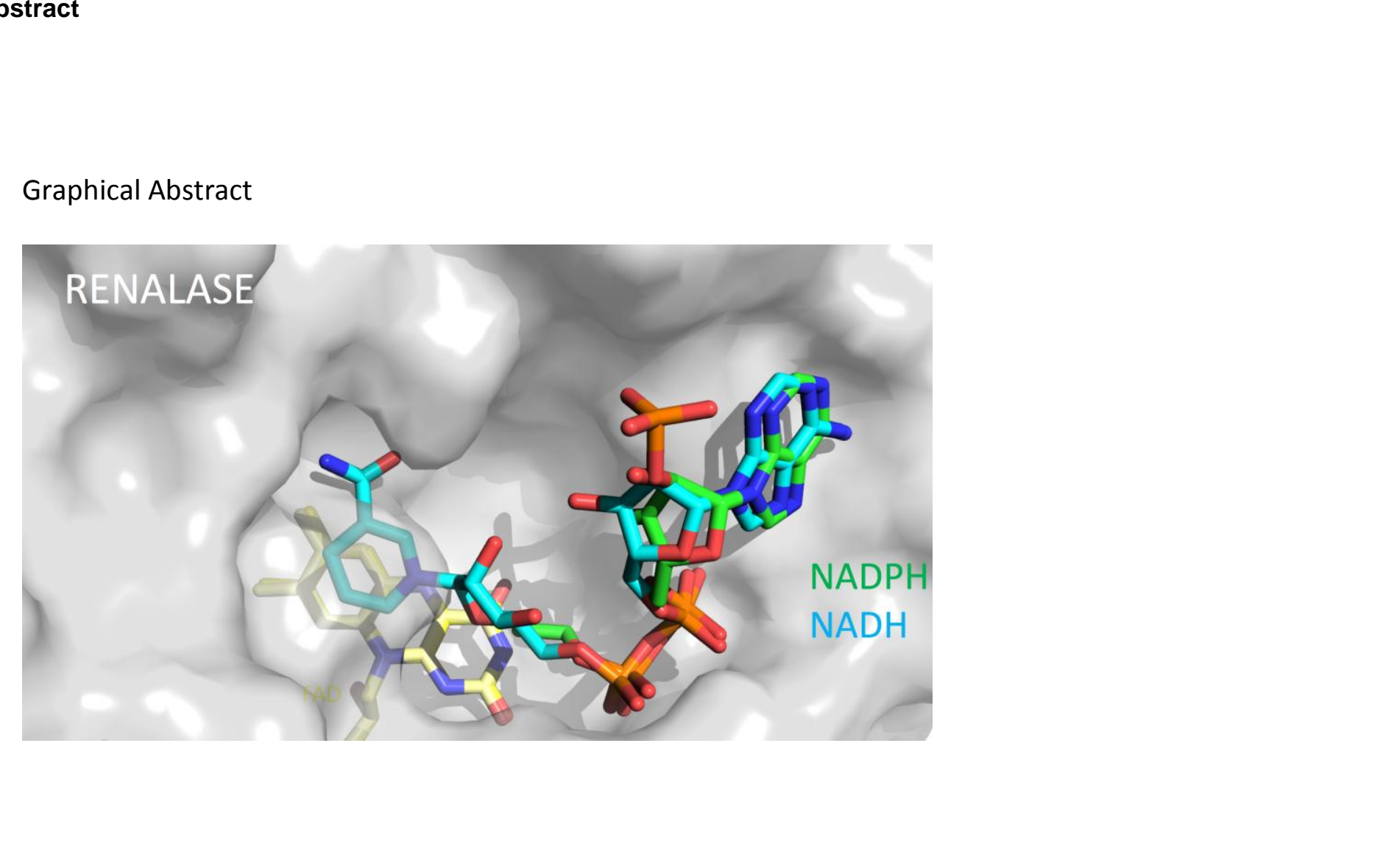